\title{
ITER Torus Diamond Window Unit: FEM Analyses and Impact on the Design
}

\author{
G. Aiello, A. Meier, T. Scherer, S. Schreck, P. Spaeh, \\ D. Strauss, A. Vaccaro \\ Karlsruhe Institute of Technology \\ Karlsruhe, Germany \\ gaetano.aiello@kit.edu
}

\author{
M. Gagliardi, G. Saibene \\ Fusion for Energy \\ Barcelona, Spain
}

\begin{abstract}
The ITER torus diamond window unit is part of the electron cyclotron (EC) upper launcher (UL) used to direct high power microwave beams generated by the gyrotrons into the plasma for heating and current drive (H\&CD) applications. The UL consists of an assembly of ex-vessel waveguides (WGs) and an in-vessel port plug (PP). The diamond window units form vacuum and confinement boundaries between the torus volume and the transmission lines (TLs) which guide beams between 1 and $1.5 \mathrm{MW}$ at $170 \mathrm{GHz}$ from the gyrotrons to the launcher.
\end{abstract}

There are eight window units attached to the assembly of the WGs, one unit for each WG. The design strategy of the unit is to have a very rigid outer frame able to withstand the potential external loads acting on the unit while thin copper cuffs brazed to the diamond disk allow indirect cooling of the disk to remove the EC power absorption during the beam transmission. The load combination given by the stringent ITER seismic level 2 (SL-2) event occurring during baking of the torus vessel is the design driver for the outer frame of the unit. In fact, the assembly of the WGs is connected from one side to the ceiling of the ITER port cell area by a support frame and to the UL PP from the other side. Movements of the torus vessel due to baking, seismic and plasma disruption events, result in forces and moments acting on the units. Furthermore, during a seismic event, the unit is subject to additional loads induced by the oscillation of the support frame attached to the ceiling. An outer frame surrounding the window unit is thus required to ensure the structural integrity and the confinement function of the unit.

This paper shows how the design of the window unit was assessed and optimized by FEM analyses. A specific methodology was developed to carry out the analyses with respect to the seismic and baking loads. At KIT, FABRY-PEROT resonators measure the loss tangent of the diamond disk and it is then used as input to the CFD and FEM analyses aiming to assess the design. The impact of the FEM analyses on the design of the window unit is discussed also together with the manufacturing aspects of the unit.

Keywords-ITER; upper launcher; diamond window unit; FEM analysis; seismic; baking.

\section{INTRODUCTION AND BACKGROUND}

The torus diamond window unit is a sub-component of the electron cyclotron heating and current drive upper launcher
(EC H\&CD UL) and it is part of the first ITER vacuum and tritium confinement system while allowing the transmission of high power microwave beams from the gyrotrons (the radio frequency sources) into the plasma. The beams have a frequency of $170 \mathrm{GHz}$ and power between 1 and $1.5 \mathrm{MW}$. The main purpose of the upper launcher is to drive local current in the plasma with the aim of suppressing neoclassical tearing modes which on one side can trigger plasma disruptions generating loads on the plasma facing components and on the other side lead to confinement degradation [1], [2].

The window unit basically consists of a $1.11 \mathrm{~mm}$ thick chemical vapor deposition (CVD) diamond disk brazed to two oxygen-free copper cuffs and this structure is then integrated into a stainless steel and nickel housing by welding. The thickness of the disk corresponds to $3 / 2$ the wavelength of the beam inside the diamond material allowing therefore fulfilling the minimum reflection condition for the beam power. The unit, being a vacuum and tritium first barrier, has thus the most stringent requirements in the ITER safety, quality, seismic, vacuum and tritium classifications [3]. It is classified as a Safety/Protection Important Component 1 (SIC/PIC) with the nuclear safety function of confinement.

Fig. 1 shows the UL consisting of an assembly of ex-vessel WGs and an in-vessel PP. The window units, together with the isolation valves, are located in the section of the WGs assembly enclosed by a support frame (called the ceiling support frame) attached to the ceiling of the ITER port cell area, as reported in Fig. 2. The ex-vessel WGs assembly is connected to the closure plate (CP) of the UL PP on the plasma side and to the TLs on the gyrotron side. The connection to the TLs is located just after the WGs support frame and it represents the interface between the procurement boundaries of the European Domestic Agency (F4E) and the American Domestic Agency (USIPO).

The design of the EC H\&CD system from this interface down to the plasma is under F4E responsibility whilst between the interface and the gyrotrons is under USIPO responsibility. It should be noted that, for purely naming convention reasons, the term WGs refer to the lines from the interface down to the PP while the term TLs refer to the ones from the interface up to the gyrotrons. 


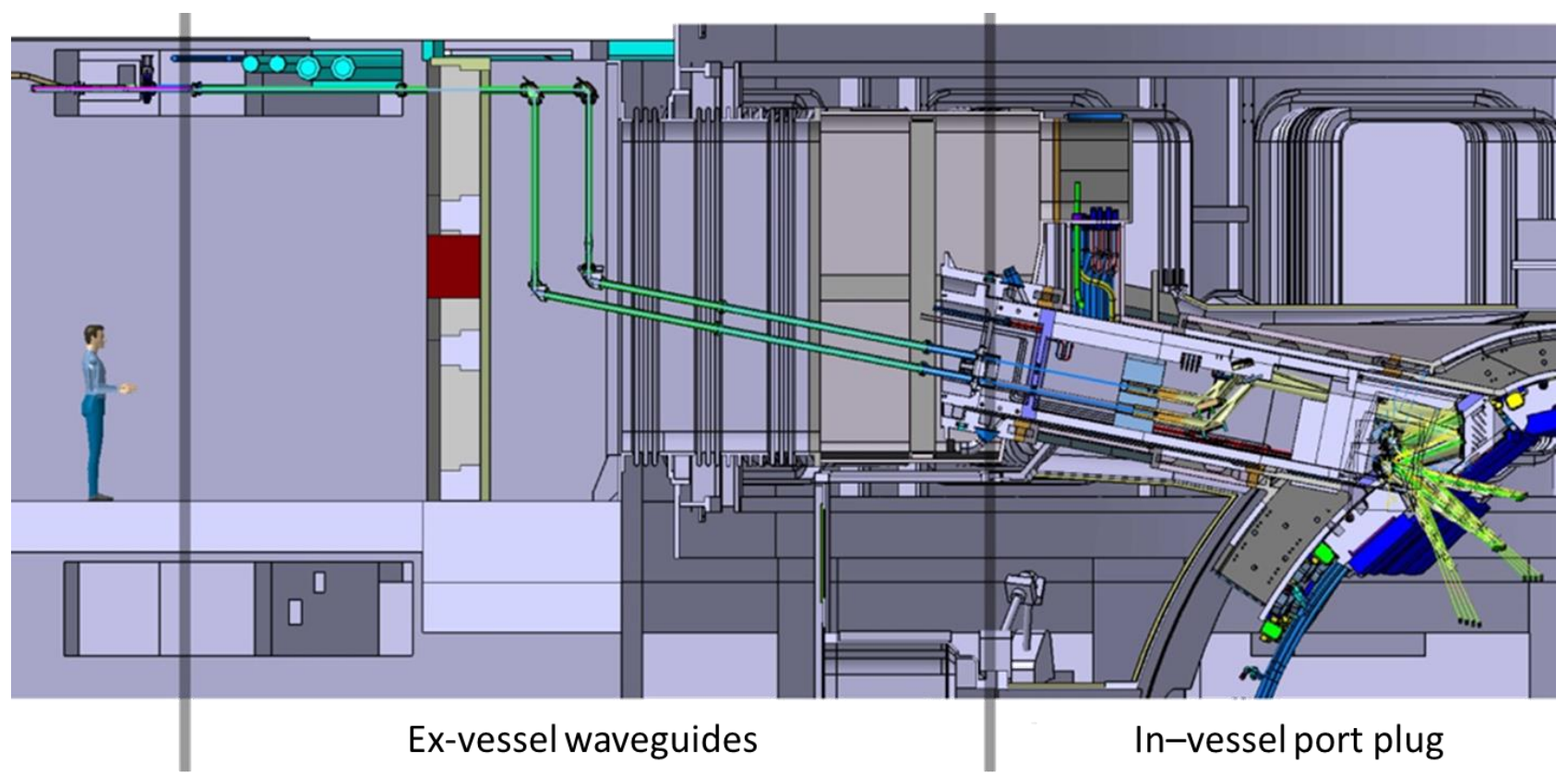

Fig. 1. Overview of the in- and ex-vessel parts of the ITER EC H\&CD upper launcher.

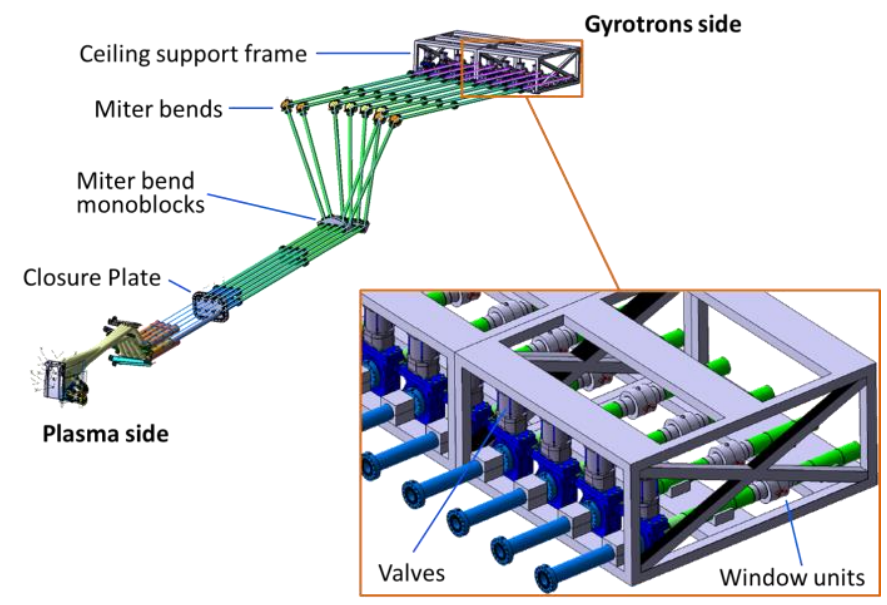

Fig. 2. Ex-vessel WGs assembly with the ceiling support frame dated at March 2014. The frame encloses isolation valves and diamond window units and it is attached to the ceiling of the port cell area.

Looking at the position of the diamond window units, it can be observed that the unit is subjected to external loads, which derive from movements of the ITER vacuum vessel (VV) of any nature (baking, seismic, plasma disruption events) and also from oscillations of the ceiling support frame in case of a seismic event. However, it has to be noted that the design of the supports for the ex-vessel WGs and the TLs is still in the development phase.

The design strategy is the following:

- To minimize as far as possible the impact of the VV displacements on the units and valves, leading thus to solutions like the support configuration shown in Fig. 2.

- To protect the sensitive inner parts (mainly the diamond disk) of the unit by a rigid outer frame able to withstand the potential external loads acting on the unit during seismic, baking, plasma disruption events and installation and maintenance phases.

In January 2014, a contract between F4E and the Karlsruhe Institute of Technology (KIT) was signed aiming at the manufacturing and qualification of prototypes of the torus diamond window unit (F4E-OPE467). Even if the design of the supports for the ex-vessel WGs is not complete yet, in the context of this contract there was thus the need to finalize the design of the unit outer frame with respect to the most stringent load combination.

A typical design of the diamond window unit is shown in Fig. 3. It consists of an ultra-low loss tangent diamond disk brazed to two copper cuffs with embedded cooling channels allowing indirect cooling of the disk. Two nickel rings, named spacer rings, connect the cuffs to corrugated stainless steel WGs which are inserted into the cuffs leaving a $100 \mu \mathrm{m}$ gap with the diamond disk. The WGs have an inner diameter of $63.5 \mathrm{~mm}$.

This specific design of the waveguide system prevents the generation of parasitic electromagnetic oscillations in the small cavities of the unit. The cooling channels are closed by external nickel rings, named cooling rings. These rings are connected among them by the stainless steel middle ring. A stainless steel outer shell surrounding the unit protects it against the external loads acting on the unit.

The design driver load combination for the outer frame of the unit is the severe SL-2 seismic event occurring during the VV baking [4]. A specific methodology was developed to carry out FEM analyses of the window unit with respect to such load combination in ANSYS Workbench [5]. The impact of such analyses on the design of the diamond window unit towards an optimum design solution is reported in this paper together with the manufacturing aspects of the unit. 

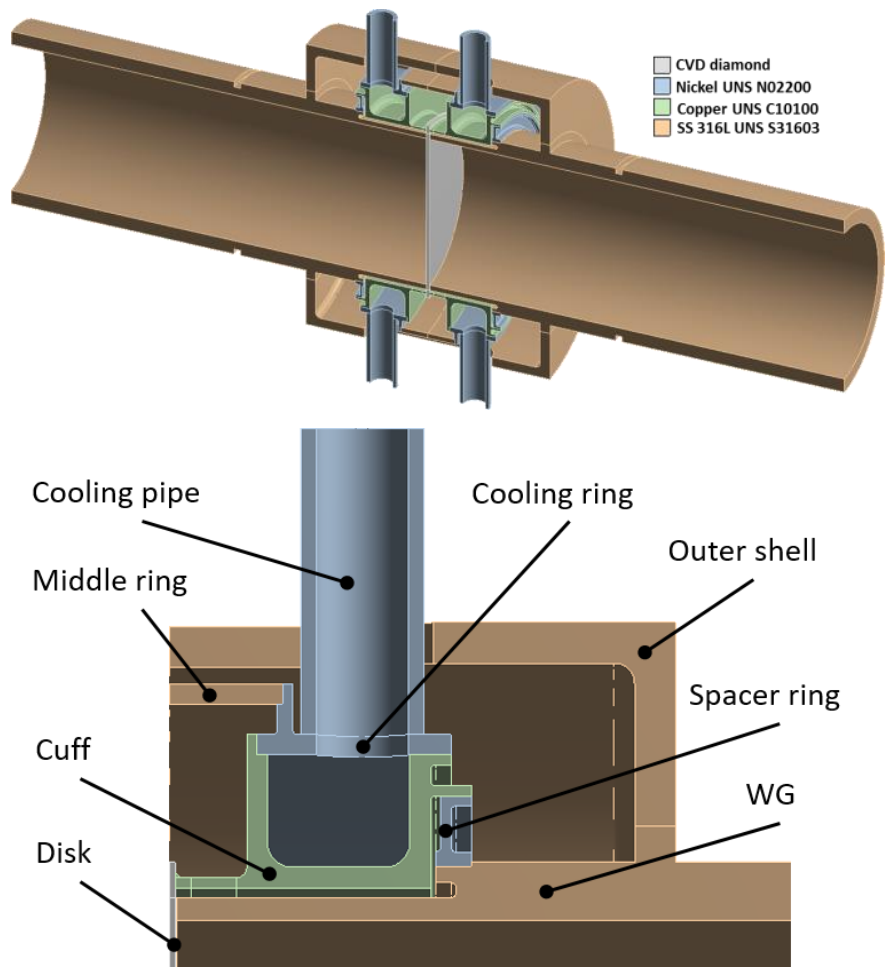

Fig. 3. Noncurrent design of the torus diamond window unit showing the design strategy with main parts and related materials. It shall be noted that a gap of $100 \mu \mathrm{m}$ exists between the disk and the WG.

Due to the EC power absorption in the disk during the beam transmission, the unit requires active cooling. As can be observed in Fig. 3, the cooling circuit is placed outside the confinement boundary of the window unit. This design choice allows separation of the coolant from the disk forming the tritium barrier and makes the unit safer, although temperatures and thermal stresses are higher than in the case of a direct cooling of the disk. CFD and FEM analyses were performed to assess the behavior of the window unit during normal operation, i.e. during the transmission of the microwave beams, and the results are also discussed in this paper. At KIT, FABRY-PEROT resonators are used to qualify the diamond disks from the perspective of the microwave properties. The loss tangent $(\tan \delta)$ is the characteristic quantity for the disk qualification and it is used as input in the analyses to calculate the power absorbed in the diamond disk.

Finally, it shall be kept in mind that the window unit has a very peculiar nature as a brittle material (the disk) is enclosed by a metallic housing and some features like the brazing are not covered by standard codes. For the most part, the window shall be thus a component qualified by experiments and some FEM analyses are only supporting analyses to the required experimental tests.

\section{MethodS}

\section{A. Analysis due to seismic and baking loads}

The FEM analysis of the window unit with respect to the seismic event SL-2 and the baking of the VV was performed in two steps as described in detail in [5]. In the first step, the loads acting on the window unit were calculated in terms of axial/shear forces and torsion/bending moments. The ex-vessel WGs assembly and the ceiling support frame, reported in Fig. 2, were modelled by line bodies while the main components (isolation valves, window units and miter bends) were modelled by point masses with the related masses and inertia moments. The geometrical section was assigned to each line body resulting in the depiction shown in Fig. 4, which also shows the connections between the WGs assembly and the support frame.

The inertial effect of the seismic event (related to the seismic vibration of the system) was investigated by the response spectrum (RS) approach that works in the frequency domain. This approach is based on the modal analysis of the system and the RS (in general, a plot of acceleration versus frequency) provides the excitation to the system. The floor response spectra (FRS) at the points UPP_flange [6] and 7316 [7] were used in the seismic analysis of the unit and they are reported in Fig. 5. The RS approach was already used in the seismic analysis of UL PP [8], thus leading to a consistent method for the seismic analysis of the whole EC H\&CD UL. The modal analysis was first performed to calculate natural frequencies and mode shapes of the system. Then, the modal results were given as input to the subsequent RS analyses. In order to obtain accurate results [9], the responses of the higher order modes (i.e. the modes falling beyond the FRS frequency range) were taken into account by a static analysis that uses the zero period acceleration (ZPA) reported in Fig. 5. In fact, beyond the FRS frequency range, the spectral acceleration is constant and there is no dynamic amplification (rigid system).
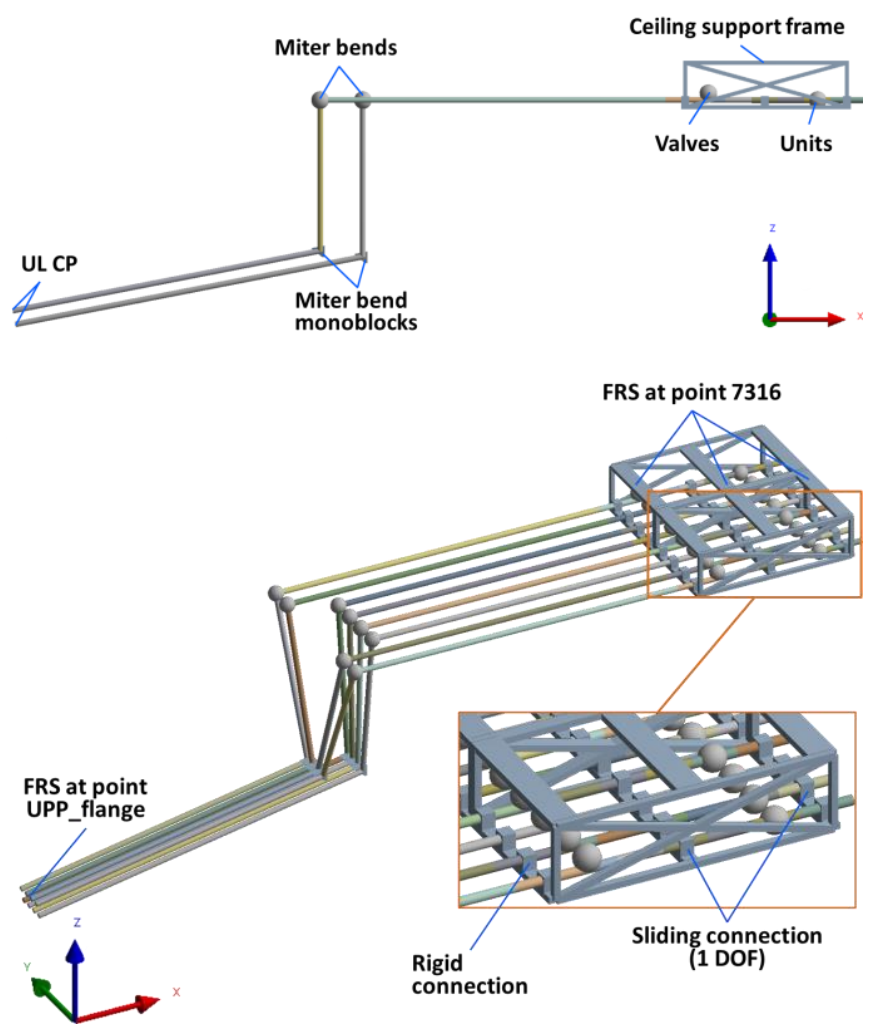

Fig. 4. FEM model of the ex-vessel WGs assembly used in the first step of the analysis for the SL-2 seismic event occurring during the VV baking. 

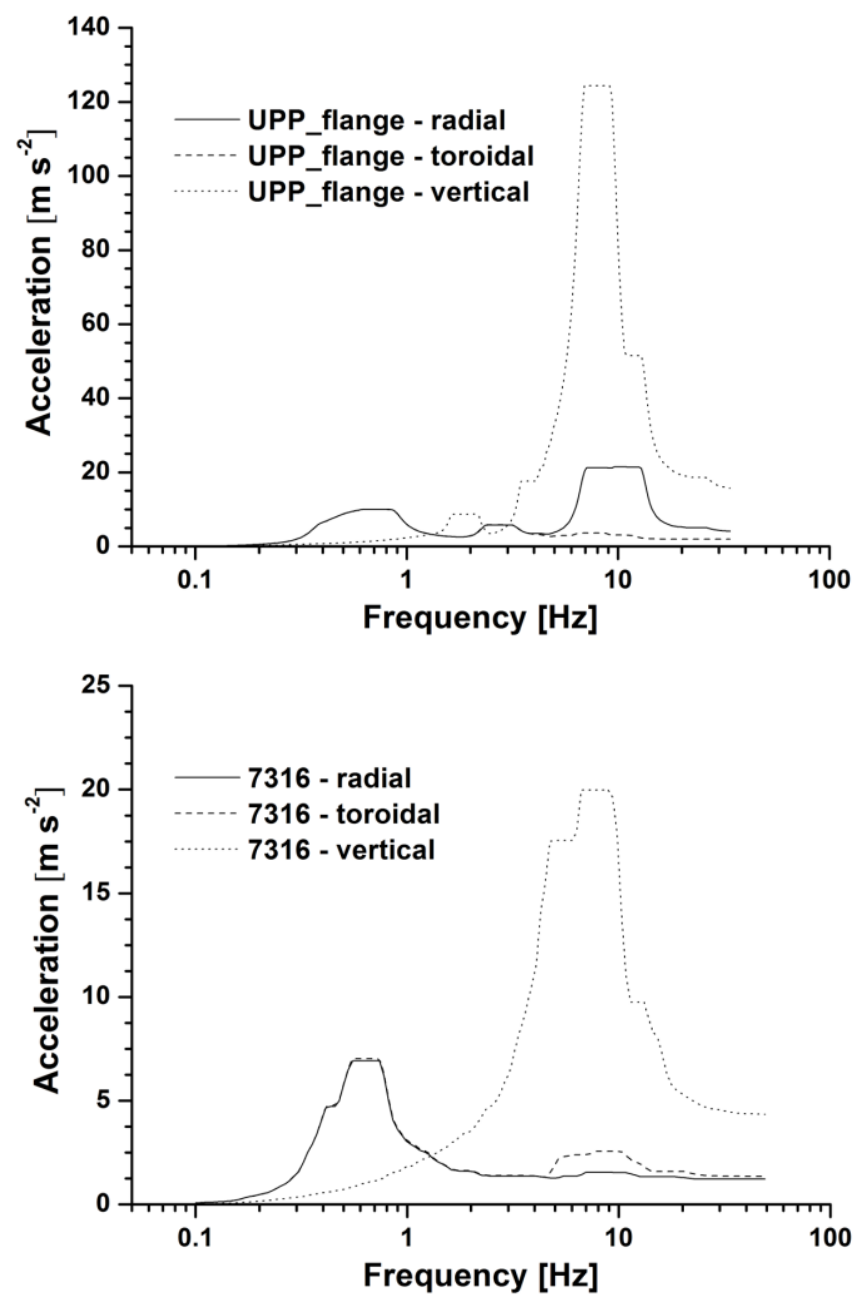

Fig. 5. FRS used as excitation in the RS analyses of the ex-vessel WGs assembly. Zero period acceleration (ZPA) in radial, toroidal and vertical directions are respectively $4.08,1.95$ and $15.7 \mathrm{~m} \mathrm{~s}^{-2}$ for the FRS at the UPP_flange point while $1.23,1.35$ and $4.34 \mathrm{~m} \mathrm{~s}^{-2}$ for the FRS at the point 7316 .

Three separate RS analyses were performed in correspondence to the three directions of excitation: radial, toroidal and vertical. In each RS analysis, the appropriate FRS and ZPA were specified. The RS analysis records only the amplitudes of the responses for each mode and therefore a combination rule among the frequency modes must be adopted to obtain the results of each RS analysis. Since the modes are not well separated in the frequency range, the complete quadratic combination (CQC) rule was used with a constant damping coefficient of $4 \%$ [10].

A further and last combination rule was applied to the results of the three RS analyses to obtain the results due to all three seismic excitations. Newmark's rule was adopted as spatial combination rule [10], [11]. The underlying assumption of this Newmark's spatial combination rule is that, when the maximum response from one earthquake component occurs, the responses from the other components are $40 \%$ of their corresponding maximum. For a given variable $\mathrm{S}$, such a rule is written as:

$$
\begin{array}{r}
S=\max \left( \pm S_{x} \pm 0.4 S_{y} \pm 0.4 S_{z} ; \pm 0.4 S_{x}\right. \\
\left.\quad \pm S_{y} \pm 0.4 S_{z} ; \pm 0.4 S_{x} \pm 0.4 S_{y} \pm S_{z}\right)
\end{array}
$$

where $S_{x}, S_{y}$ and $S_{z}$ are the maximum responses of that variable (e.g., vertical force) respectively due to the seismic excitation in radial, toroidal and vertical direction, while $\mathrm{S}$ is the resulting maximum response due to all three excitations. Due to the nature of the RS analysis, $S_{x}, S_{y}$ and $S_{z}$ are positive values and thus the rule is reduced to only 3 of the initially foreseen 24 combinations.

The kinematic effect of the seismic event (related to the relative motion between the two locations of the FRS in Fig. 4) was investigated by a structural analysis where the seismic displacements were applied to the UL CP. The displacements provided at the point VV_D1 [6] of interest for the seismic analysis of the unit were used. They are the VV relative displacements to the ITER Basemat and amount to $\Delta \mathrm{x}=6.29$ $\mathrm{mm}, \Delta \mathrm{y}=4.6 \mathrm{~mm}$ and $\Delta \mathrm{z}=5.84 \mathrm{~mm}$ respectively in the radial, toroidal and vertical direction.

The effect due to VV baking was investigated by another structural analysis where the displacements were applied to the UL CP. The displacements were obtained by carrying out the difference between the displacements given for baking and normal operation in [12] and they amount to $\Delta \mathrm{x}=16.5 \mathrm{~mm}, \Delta \mathrm{y}$ $=0 \mathrm{~mm}$ and $\Delta \mathrm{z}=24.4 \mathrm{~mm}$ respectively in radial, toroidal and vertical direction.

The analysis results were obtained in terms of distributions of axial/shear forces and torsion/bending moments acting on the eight diamond window units. For the two seismic effects, the distributions of the loads were taken with \pm sign variation as the system oscillates during the seismic event. For the effect due to the VV baking, in this case, the loads have a unique sign as the VV moves only outward and upward during the baking event.

Summing up the several distributions of loads obtained by the analyses with the sign that produces the maximum load on the unit, the final distributions acting on the window units were obtained for the load combination SL-2 event during the VV baking. The maximum loads of the final distributions acting on the eight units were considered in the second step of the analysis.

In this second step, the calculated loads were applied to a detailed FEM model of the window unit to calculate stresses and displacements as shown in Fig. 6. The dead weight of the unit was also considered. Several structural analyses of the window unit were performed in order to find the optimum design solution for the unit outer frame, taking into account also the need of a second tritium barrier and a real-time monitoring of all interspaces inside the unit. 


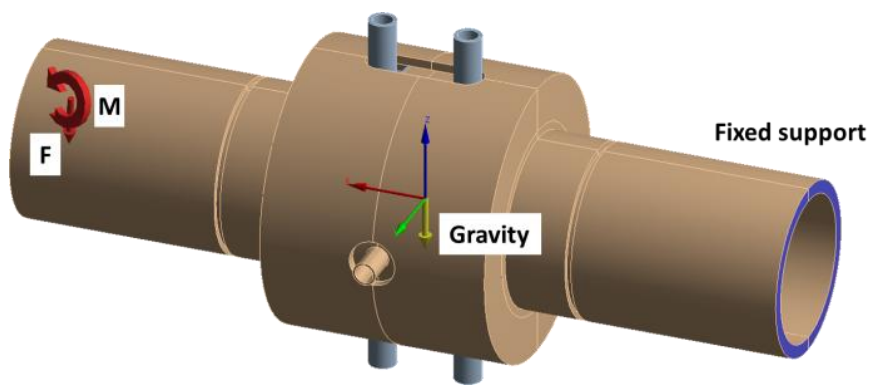

Fig. 6. Noncurrent design of the torus diamond window unit showing the loads and boundary conditions applied in the second step of the analysis for the SL-2 seismic event occurring during the VV baking.

\section{B. Analysis due to EC power absorption}

The analysis aiming to assess the behavior of the window unit during normal operation was undertaken in two steps. First a CFD conjugated heat transfer analysis (both fluid and solid domains are modelled) was performed to calculate the temperature distribution and the pressure drop. In the second step, the temperature distribution together with the other operational loads was given as input to a structural analysis to calculate the resulting stresses in the unit.

A quarter of the window unit was considered in the analysis because of the symmetry. In addition, only for the CFD conjugated analysis, the model contains the fluid (water), the diamond disk and the copper cuff as the other parts do not contribute to the cooling of the disk. A $1 \mathrm{MW} \mathrm{HE}_{11}$ mode beam was taken into account in the analysis and the power absorbed in the disk was calculated according to:

$$
P_{a b s}=P_{\text {beam }} \cdot \frac{f}{c} \cdot \pi \cdot\left(1+\varepsilon_{r}\right) \cdot \tan \delta \cdot t
$$

where $P_{a b s}$ is the absorbed power in $\mathrm{W}, P_{\text {beam }}$ is the beam power, $f$ is the beam frequency, $\varepsilon_{r}$ is the dielectric constant of diamond, $\tan \delta$ is the loss tangent of diamond and $t$ is the thickness of the disk. The $\tan \delta$ is provided by the experimental measurements performed at KIT with the FABRY-PEROT resonators (spherical and hemi-spherical) on the diamond disks fabricated in the prototyping activity of the unit. The measurements at $170 \mathrm{GHz}$ led to $\tan \delta$ values lower than $2 \times 10^{-5}$ for $50 \%$ fraction of the inspected area of the disk. Considering $P_{\text {beam }}=1 \mathrm{MW}, f=170 \mathrm{GHz}, \varepsilon_{r}=5.67, \tan \delta=2 \times 10^{-5}$ and $t=$ $1.11 \mathrm{~mm}$, the power absorbed in the disk resulted in $264 \mathrm{~W}$.

The heat generation load was applied to the disk by the Bessel function of order zero which describes the power pattern of the $\mathrm{HE}_{11}$ mode beam inside the WG:

$$
q^{\prime \prime \prime}(r)=A \cdot\left[J_{0}(x)\right]^{2}, x=2.405 \cdot \frac{r}{a}
$$

where $q^{\prime \prime \prime}$ is the volumetric power density in $\mathrm{W} \mathrm{m}^{-3}, A$ is the normalizing constant, $r$ is the radial coordinate and $a=31.75$ $\mathrm{mm}$ is the inner radius of the waveguide. The power density distribution $q^{\prime \prime \prime}$ was normalized in order to obtain the calculated value of absorbed power in the disk. A volumetric flow rate of $20 \mathrm{l} / \mathrm{min}$ at $20{ }^{\circ} \mathrm{C}$ was applied to the complete window unit (there are two inlets, it is assumed $10 \mathrm{l} / \mathrm{min}$ in each inlet). After running the CFD conjugated analysis, the temperature distribution was transferred to the static structural analysis to calculate the resulting stresses. In this analysis, the dead weight of the unit, the pressure of the water in the cooling circuit (9 bar absolute) and the external atmospheric pressure ( 1 bar) were also applied to the model.

\section{RESULTS AND DISCUSSION}

Fig. 7 reports a typical plot of the distributions of loads on the eight window units obtained by the first step of the analysis for the design driver load combination SL-2 event during the VV baking. Distributions of axial/shear forces and torsion/bending moments acting on the units were obtained. The maximum of such load distributions acting on the eight units is shown in TABLE I. The order of magnitude of the loads on the units is very low thanks to the stiff ceiling support frame of the ex-vessel WGs. In previous design versions of the ex-vessel WGs assembly and their supports, the window unit was subject to very high loads (hundreds of $\mathrm{N}$ and $\mathrm{N} \mathrm{m}$ ) for the SL-2 event during the VV baking. This resulted in the robust design shown in Fig. 8a. An outer frame with two walls attached to the WGs was necessary to withstand the high loads acting on the unit, significantly increasing the complexity of the unit manufacturing and assembling.

The new loads reported in TABLE I allowed simplifying this outer frame leading to a unit design more compact and feasible to manufacture. The loads mainly cause stresses in the nickel spacer rings and reduce the $100 \mu \mathrm{m}$ gap between the disk and the stainless steel WG. The nickel spacer rings and the gap were thus the critical regions observed in the structural analyses of the unit. As a reference, it has to be kept in mind that the allowable stress limit $\mathrm{S}_{\mathrm{m}}$ is around $70 \mathrm{MPa}$ for the nickel $\mathrm{N} 02200$ in the form of a bar/rod from $-30^{\circ} \mathrm{C}$ up to $300^{\circ} \mathrm{C}$, according to the ASME Section II - Part D [13].

Fig. 8 shows the evolution of the unit design. First, the outer shell was reduced to a single wall shell of $4 \mathrm{~mm}$ (Fig. $8 \mathrm{~b}$ ) leading to a maximum equivalent stress of $73 \mathrm{MPa}$ in the spacer rings and a gap reduction of $12 \mu \mathrm{m}$. Despite the robust outer frame of the unit in Fig. 8a, the old loads were responsible for a stress in the rings greater than $150 \mathrm{MPa}$, well above the allowable limit. Then, replacing two $90^{\circ}$ angled sides by one $45^{\circ}$ angled side in the outer shell (Fig. 8c), the stress was further decreased to $45 \mathrm{MPa}$ and the gap reduction limited to $8 \mu \mathrm{m}$. Finally, the need for a second tritium barrier and a real-time monitoring of all interspaces inside the unit led to the optimized design shown in Fig. 8d.

In the previous design variants, the outer frame was formed by only the outer shell surrounding completely the unit. There were holes in the outer shell due to the passage of the cooling pipes (Fig. 3) and thus failures of the joints in the unit would have allowed tritium to get into the port cell area.

The solution was to have an outer frame formed by the middle rings and cooling rings with increased thickness and by outer shells acting as a second tritium barrier in case of failures of the joints in the unit. In addition, vacuum bridges (holes) were accommodated in the cooling rings among the interspaces of the unit to allow their real-time monitoring by the diagnostic pipes (increased from two to four) attached to the middle rings. 


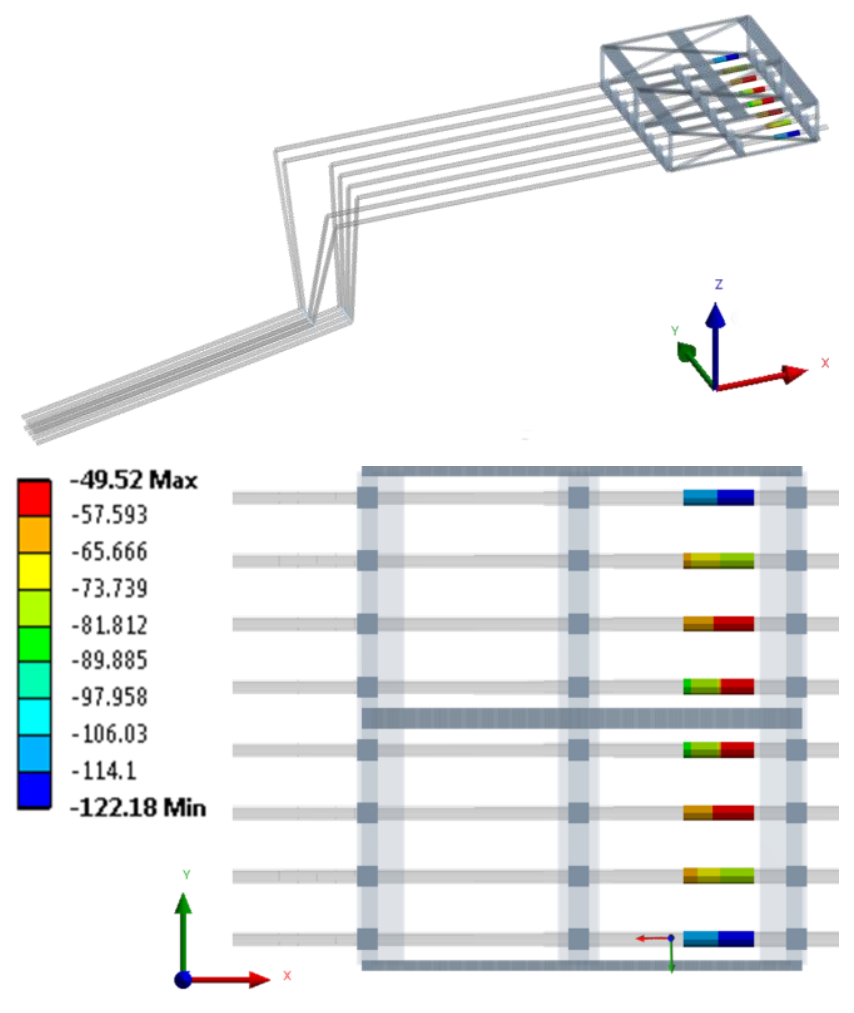

Fig. 7. Distribution of the vertical force along the eight window units due to the load combination SL-2 event occurring during the VV baking. Values are in $\mathrm{N}$ and refer to the local reference system placed close to the units.

TABLE I. MAXIMUM LOADS ACTING ON THE EIGHT WINDOW UNITS WITH RESPECT TO THE LOCAL REFERENCE SYSTEM OF THE UNITS SHOWN IN FIG. 7.

\begin{tabular}{|c|c|c|}
\hline Loads & Type of loads & Maximum values \\
\hline Fx $[\mathrm{N}]$ & Axial force & 12.3 \\
\hline Fy $[\mathrm{N}]$ & Horizontal force & -19.8 \\
\hline Fz $[\mathrm{N}]$ & Vertical force & -122.2 \\
\hline $\mathrm{Mx}[\mathrm{N} \mathrm{m}]$ & Axial torsion & 16.3 \\
\hline $\mathrm{My}[\mathrm{N} \mathrm{m}]$ & Horizontal bending & 34.7 \\
\hline $\mathrm{Mz}[\mathrm{N} \mathrm{m}]$ & Vertical bending & 6.3 \\
\hline
\end{tabular}

This solution made the design of the unit more compact and even stiffer. In fact, the maximum equivalent stress in the spacer rings was further decreased to the range 20-24 MPa (Fig. 9), well below the allowable limit, and the gap between the disk and the WG reduced by only $6 \mu \mathrm{m}$. The maximum principal stress in the diamond disk is lower than $1 \mathrm{MPa}$, showing thus that the sensitive inner parts are not affected by the external loads acting on the unit.

The optimum design solution of the window unit obtained by the FEM analyses is reported in Fig. 10 together with the exploded view showing the several parts to be manufactured and the proposed assembling sequence of the unit. The window unit consists of 13 parts and it is foreseen to assemble them by using only two types of joints: brazing between disk and cuffs and electron beam (EB) welding between all other parts. The brazing material shall be a copper-silver-titanium (CuAgTi) alloy. Titanium creates a good connection with the diamond disk surface as it has a strong affinity with carbon atoms. Fig. 10a shows that there are $6 \times 2$ symmetric parts plus the diamond disk forming the unit. The two inlet and outlet cooling pipes are fabricated together with the cooling rings while the four diagnostic pipes are manufactured together with the middle rings.

The total number of joints amounts to 18 , in particular to $9 \times 2$ because of the symmetry in the unit. Looking at the Fig. $10 \mathrm{~b}$, the cuffs are first welded to the cooling rings (box no. 1) and then the brazing between the disk and the cuffs is performed (box no. 2) leading to the first subsystem of the unit. In parallel, the WGs are welded to the spacer rings (box no. 3) leading to the second subsystem of the unit. The two subsystems are then joined together by carrying out the weld between the cuffs and the spacer rings shown in the box no. 4 . Finally, the outer shells are welded to the assembly (box no. 5) and last the middle rings are added (box no. 6).

The most challenging joint of the unit is the brazing of the disk to the cuffs, which is carried out in a vacuum oven at about $800^{\circ} \mathrm{C}$ and then the temperature of the brazed parts (disk and cuffs) is decreased down to room temperature. In fact, as diamond and copper have very different thermal expansion coefficients, high stresses build up at the interface diamondcopper during the cool down phase. The metallic parts of the unit are joined by EB welding as this process allows minimization of the deformations induced in the unit by the welded joints. High welding deformations might lead to the failure of the disk. Therefore, with respect to other welding techniques, the use of the EB welding process makes the structural integrity of the diamond disk safer during the assembling of the unit.

The temperature distribution generated in the unit during the transmission of a 1 MW beam is shown in Fig. 11. It should be reminded that only the water, the disk and the cuff were modelled as the other parts do not give any contribution to the cooling. In fact, it can be observed that the temperature in the cuff immediately reaches the inlet temperature of the fluid (20 $\left.{ }^{\circ} \mathrm{C}\right)$. As expected, the maximum temperature is located at the center of the diamond disk and it is lower than $60{ }^{\circ} \mathrm{C}$, well below the temperature limit for diamond $\left(250-300{ }^{\circ} \mathrm{C}\right)$. The analysis also confirmed that the pressure drop in the cooling circuit of the window is very low, being limited to only 5.7 $\mathrm{kPa}$.

Finally, Fig. 12 and Fig. 13 show respectively the stress distributions resulting in the metallic parts of the unit and the diamond disk during normal operation. The equivalent stress is in the range $30-66 \mathrm{MPa}$ at the interface of the cuff with the diamond disk while it is in the range $1-18 \mathrm{MPa}$ away from this interface. These thermal stresses can be safely accepted as, performing the check for ratcheting, they are well below the allowable limit of $111 \mathrm{MPa}$ for pure copper (3Sm rule with $\mathrm{S}_{\mathrm{m}}$ $=37 \mathrm{MPa}$ at $20^{\circ} \mathrm{C}$ for copper in the form of a rod [14]). The maximum principal stress in the disk is below $30 \mathrm{MPa}$ and it can be also safely accepted since the permissible stress for diamond is $150 \mathrm{MPa}$ (ultimate stress is $450-500 \mathrm{MPa}$ [15]). 


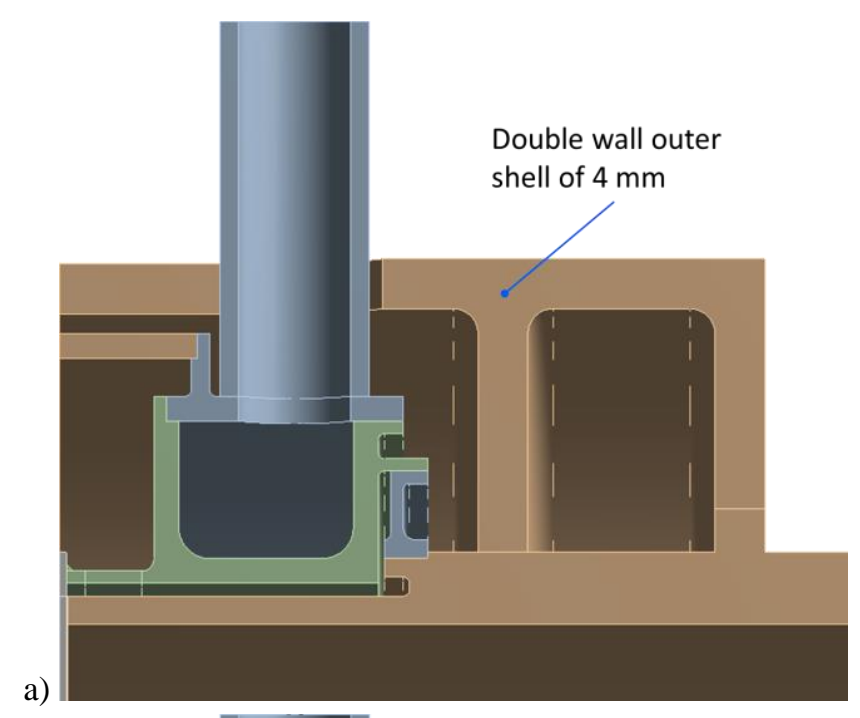

a)

c)

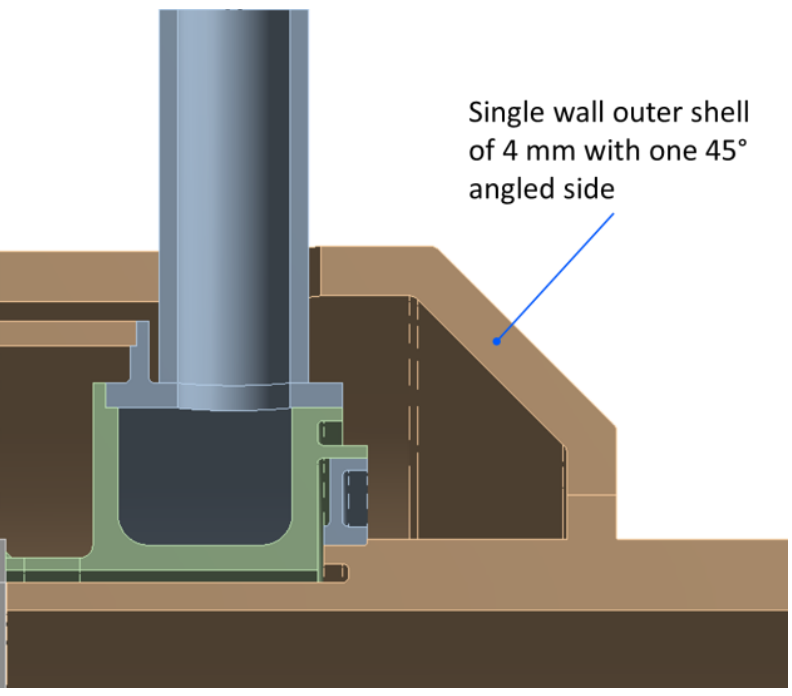

b)

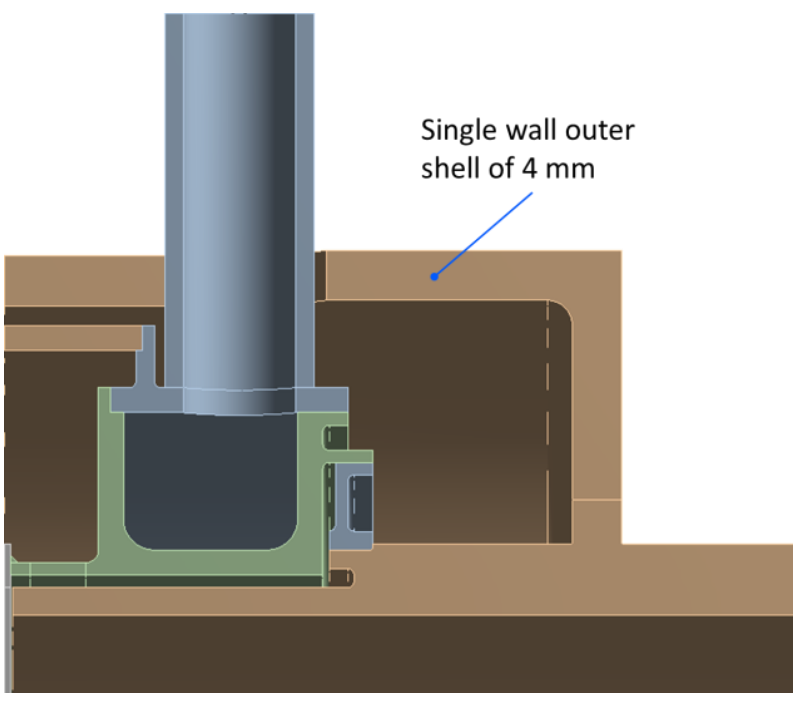

Middle and cooling rings form the outer frame of the unit together with the outer shells

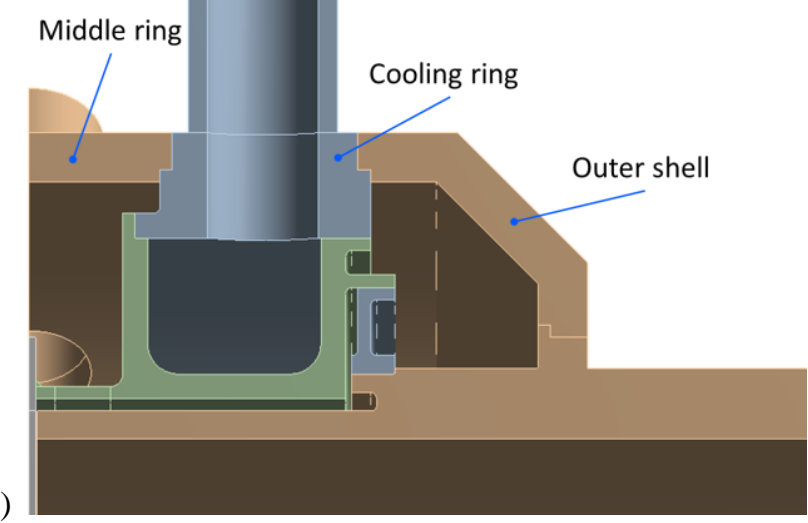

Fig. 8. Design variants of the window unit outer frame investigated with respect to the design driver load combination SL-2 event occurring during the VV baking. The loads of the TABLE I allowed simplifying the robust design of the unit reported in (a). An optimum design solution was achieved leading to the current design of the window unit shown in (d).

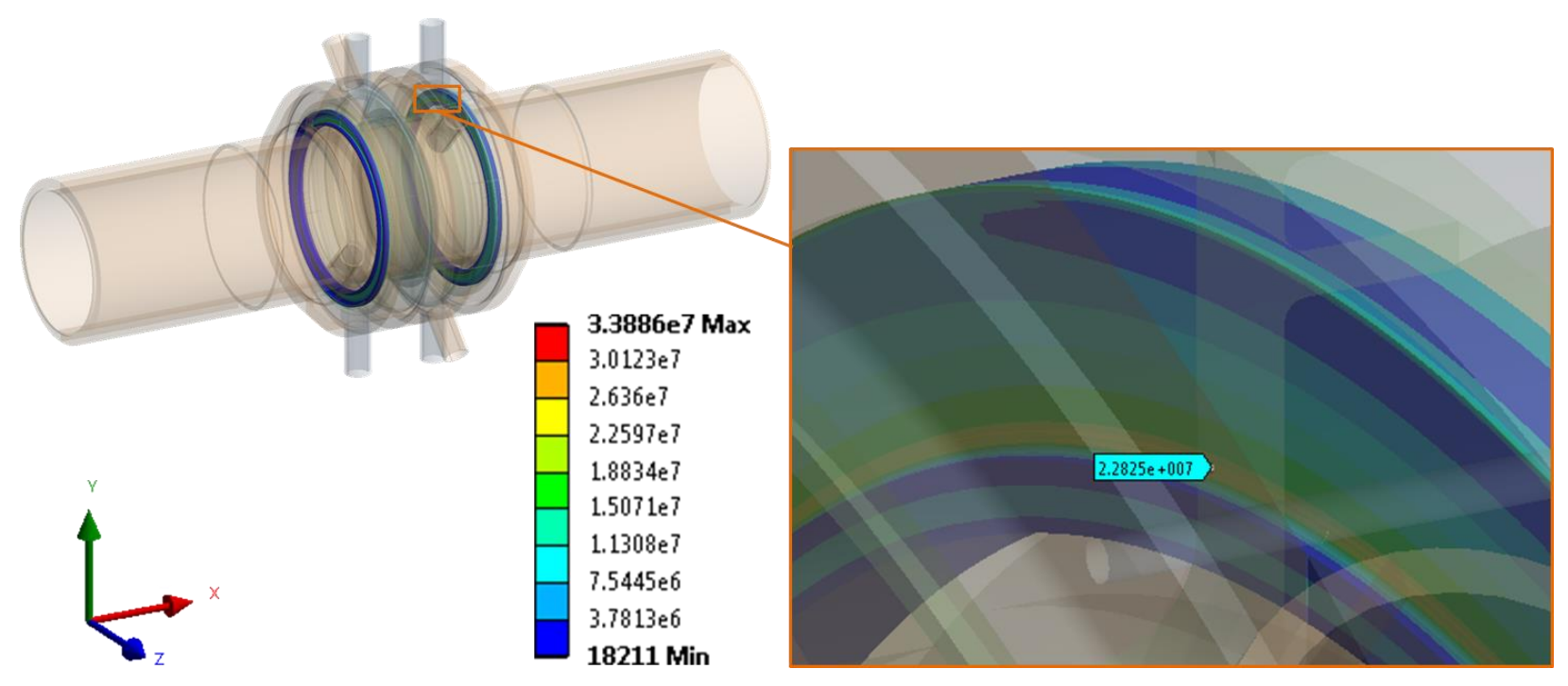

Fig. 9. Equivalent stress distribution in the nickel spacer rings of the optimum design variant shown in Fig. 8d. Values are in Pa. 

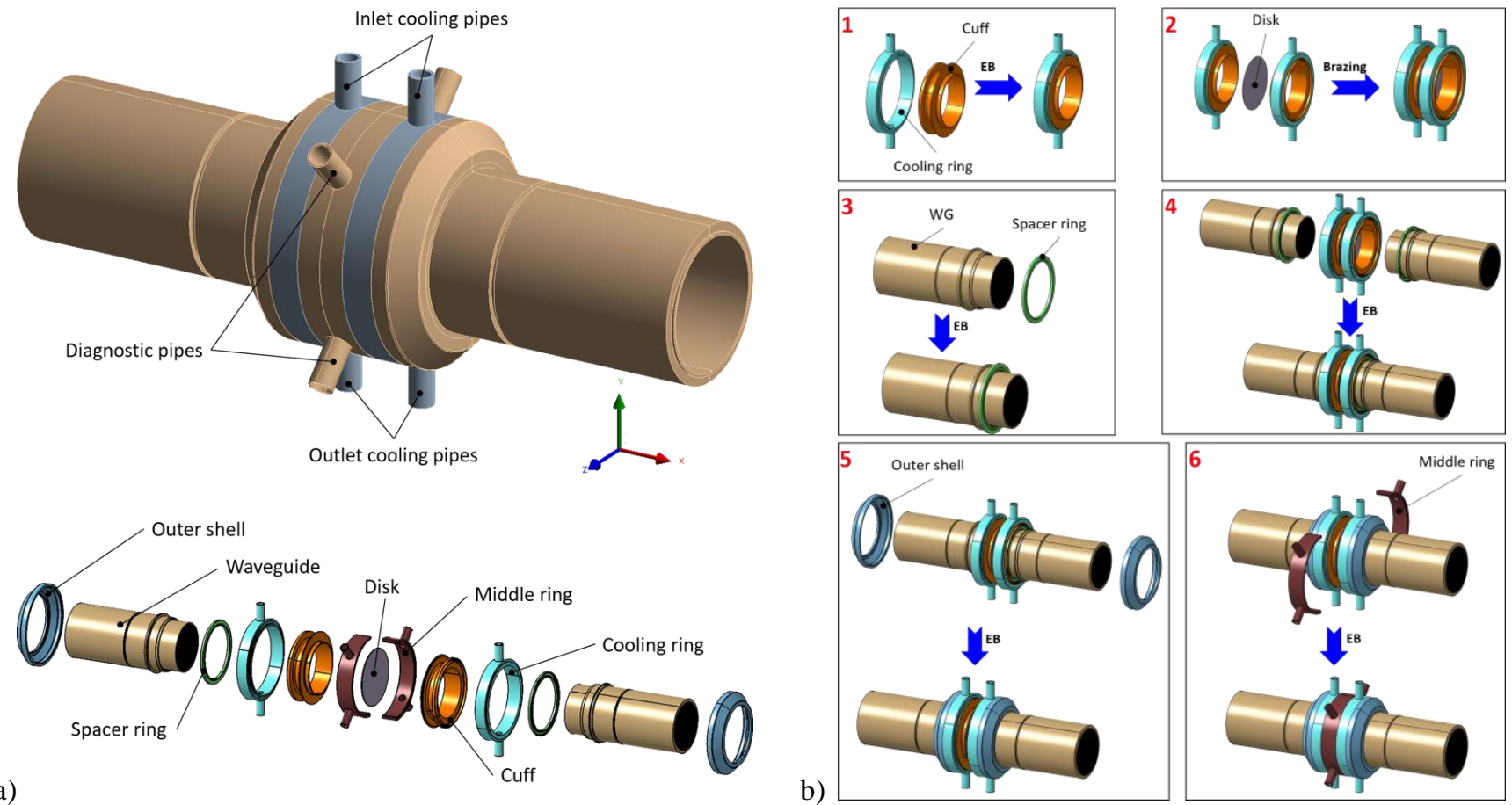

Fig. 10. Exploded view (a) and assembling sequence (b) of the diamond window unit optimized by FEM analyses. The several parts to be manufactured and the type of joints to be implemented are indicated.

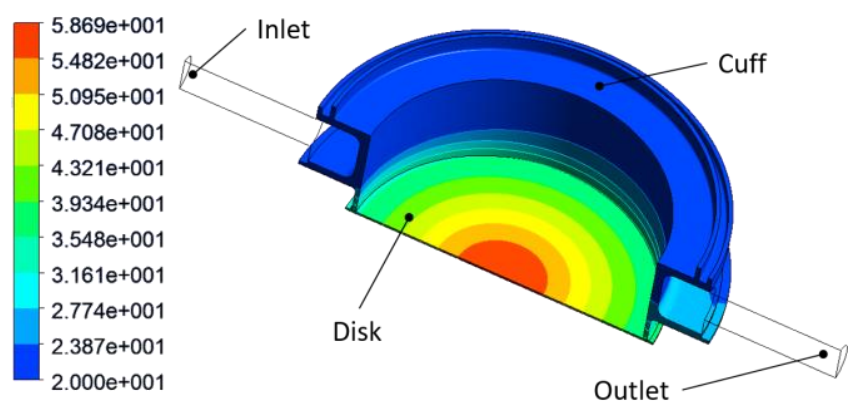

Fig. 11. Temperature distribution in the window unit resulting from the CFD conjugated heat transfer analysis. Values are in ${ }^{\circ} \mathrm{C}$.
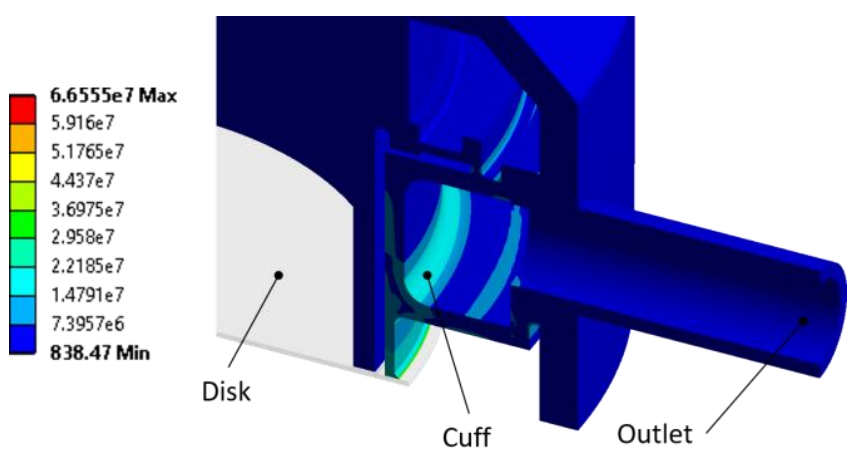

Fig. 12. Equivalent stress distribution in the window unit during normal operation. Values are in Pa.

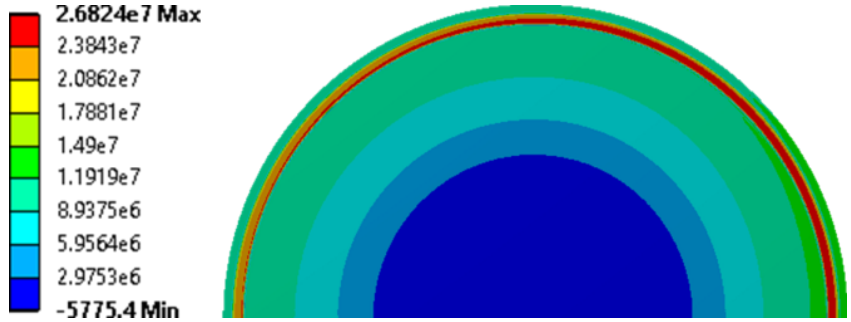

Fig. 13. Maximum principal stress distribution in the diamond disk during normal operation. Values are in Pa.

However, the main failure mode considered for the diamond disk is the failure to fracture. The design driver of the disk is represented by the cyclic pressure loads acting on the disk throughout the commissioning and operating phase of the EC system. Successful cyclic pressure fatigue tests (including the final test with 2 bar pressure difference across the disk) were carried out at KIT on brazed disk mock-ups with a disk diameter of $80 \mathrm{~mm}$ (corresponding to the WG inner diameter of $63.5 \mathrm{~mm}$ ) and thickness of $1.11 \mathrm{~mm}$ [16].

\section{CONCLUSIONS AND OUTLOOK}

The torus diamond window unit is a crucial component in the EC H\&CD UL acting as first confining tritium and vacuum barrier. It is a very unique component as it contains a brittle material (diamond) arranged in a metallic structure, it requires non-standard industrial joining technique like the brazing between brittle material and ductile material, it requires joining techniques able to release a very low amount of energy during the welding processes and it requires a design able to guarantee no stress propagation towards the inner sensitive parts, in particular the diamond disk. 
An outer frame in the unit is thus required against the external loads that might affect its nuclear safety function of confinement. An optimum design of the window unit was achieved by FEM analyses against the design driver load combination, which is the seismic event SL-2 occurring during the baking of the vacuum vessel. This optimum design also takes into account the need of a second tritium barrier and a real-time monitoring of all interspaces inside the unit. Furthermore, during the transmission of the microwave beams, this design of the window unit leads to temperatures and stresses well below the limits.

Finally, the manufacturing of the unit was discussed by showing the proposed assembling sequence. The next steps are the manufacturing and the qualification of window unit prototypes in compliance with the requirements of the ASME Boiler and Pressure Vessel Code Section III - Subsection NC [13] and also of a dedicated testing program, which shall be developed along with the prototyping activity.

\section{ACKNOWLEDGMENT}

This work was supported by Fusion for Energy under the grant contract No. F4E-2010-GRT161 and the service contract No. F4E-OPE-467. The views and opinions expressed herein reflect only the author's views. Fusion for Energy is not liable for any use that may be made of the information contained therein.

\section{REFERENCES}

[1] D. Strauss, G. Aiello, A. Bruschi, R. Chavan, D. Farina et al., "Progress of the ECRH upper launcher design for ITER," Fusion Eng. Des., vol. 89, pp. 1669-1673, 2014.

[2] M. Henderson, "EC safety system philosophy," ITER IDM: BN7UPS v1.2, January 2013.
[3] M. Henderson, "EC UL quality classification," ITER IDM: 3CNFEC v2.1, July 2011.

[4] M. Gagliardi and M. Henderson, "Load specification for the EC upper launcher," F4E IDM: 25QD28 v3.0, January 2015.

[5] G. Aiello, A. Meier, T. Scherer, S. Schreck, P. Spaeh et al., "The ITER EC H\&CD upper launcher: methodology in the FEM analyses of the diamond window unit subject to seismic and baking loads," Proceedings of the $26^{\text {th }}$ Symposium on Fusion Engineering, 31 May -4 June 2015, Austin, TX, USA.

[6] G. Mazzone, V. Sorin, and A. Alekseev, "Global Tokamak seismic analysis report," ITER IDM: 33W3P4 v2.1, July 2011.

[7] X. Bailo Fernandez, "Floor response spectrum in the Tokamak building," F4E IDM: 256Y3K v1.0, March 2012.

[8] G. Aiello, A. Vaccaro, D. Combescure, R. Gessner, G. Grossetti et al., "The ITER EC H\&CD upper launcher: seismic analysis," Fusion Eng. Des., vol. 89, pp. 1809-1813, 2014.

[9] L. Patisson, A. Buet, and P. Stewart, "ITER structural design code for buildings (I-SDCB) - part 1: design criteria," ITER IDM: 283B24 v2.10, June 2012.

[10] Translation of Seismic Nuclear Safety Authority Guidelines ASN/Guide/2/01 "Taking seismic risk into consideration for nuclear facility and civil works design,” ITER IDM: 25EUYG v1.0, 2007.

[11] J. Nie, R.J. Morante, M. Miranda, and J. Braverman, "On the correct application of the 100-40-40 rule for combining responses due to three directions of earthquake loading," Proceedings of the ASME 2010 PVP conference, Bellevue, Washington, 2010.

[12] M. Cambazar and J.M. Martinez, "VV global structural analysis with dual hinge VV supports," ITER IDM: 3T85AQ v2.0, June 2011.

[13] The American Society of Mechanical Engineers: https://www.asme.org/.

[14] V. Barabash, "Appendix A to SDC-IC, Materials design limit data," ITER IDM: 222RLN v3.3, January 2013.

[15] M. Thumm, "State of the art of high power gyro-devices and free electron masers," KIT Scientific Reports 7575, 2010.

[16] S. Schreck, G. Aiello, N. Casal, V. Gracia, M. Henderson et al., "Pressure tests supporting the qualification of the ITER EC H\&CD upper launcher diamond window," Fusion Eng. Des., in press, https://doi.org/10.1016/j.fusengdes.2018.11.002. 